\title{
Uma aplicação de algoritmos genéticos simples e compacto para estimação de componentes harmônicas
}

\author{
André Luís da Silva Pessoa ${ }^{1}$ \\ Pedro Henrique da Costa Ulisses ${ }^{1}$ \\ Hermes Manoel Galvão Castelo Branco ${ }^{1}$ \\ Ricardo de Andrade Lira Rabêlo ${ }^{2}$
}

\begin{abstract}
Resumo: Este trabalho apresenta uma abordagem para o problema da estimação de componentes harmônicas presente nos sistemas elétricos de potência, utilizando algoritmos evolutivos. Os dados de referência para o trabalho foram obtidos por meio do software Alternative Transients Program (ATP). Foram aplicados o algoritmo genético compacto e o algoritmo genético simples para estimar os parâmetros a fim de obter uma onda o mais próximo possível da onda fornecida pelo ATP. Os resultados obtidos com os algoritmos evolutivos citados são comparados entre si, em diversos cenários, e com os valores obtidos com a onda de referência gerada pelo software ATP. As comparações foram feitas com o intuito de evidenciar qual algoritmo resolve o problema exposto para uma aplicação dispondo de recursos computacionais limitados. Com base nos resultados, verificou-se que o algoritmo genético compacto resolve de forma satisfatória o problema proposto, sendo o CGA mais indicado que o SGA em situações que necessitam de um menor esforço computacional.
\end{abstract}

Palavras-chave: Algoritmo genético compacto. Algoritmo genético simples. Harmônicas. Sistemas elétricos de potência.

\begin{abstract}
The following work presents an approach for the harmonic components estimation problem, present on electrical power systems, by making use of evolutionary algorithms. The referential data was obtained by the alternative transients program (ATP) software. Compact and simple genetic algorithms where then applied to estimate the parameters to generate a wave as similar as possible to the one provided by the ATP software. The results yielded by the aforementioned evolutionary algorithms were then compared with one another in a number of scenarios, and using the values obtained by the wave of reference generated by the ATP software. The comparisons were used to seek evidence of which algorithm solved the problem, in a setting with limited availability of computational resources. Based on the generated results, it has been found that the Compact Genetic Algorithm satisfactorily solves the proposed problem, and it is the most indicated method, for when less computational effort is required.
\end{abstract}

Keywords: Compact genetic algorithm. Electrical power systems. Harmonics. Simple genetic algorithm.

\section{Introdução}

Idealmente, os sistemas elétricos de potência (SEPs) devem apresentar uma rede simétrica [1] e operar com forma de onda da tensão e corrente o mais próximo possível de uma senoide, contendo magnitude constante, bem como a frequência de $60 \mathrm{~Hz} \pm 0.5 \mathrm{~Hz}$. Porém, essa situação nem sempre é encontrada, o que implica na ocorrência de distúrbios na qualidade da energia elétrica $(Q E E)$ [2]. Os problemas na $Q E E$ estão associados a qualquer distúrbio manifestado na tensão, corrente ou desvio de frequência que resulte em falha ou operação indevida dos

${ }^{1}$ UESPI, Campus Poeta Torquato Neto - Piauí (PI) - Brasil.

\{andreluissilvapessoa, ulissesph, hermesmanoelegmail.com\}

${ }^{2}$ UFPI, Campus Ministro Petrônio Portella - Piauí (PI) - Brasil.

\{ricardor_usp@yahoo.com.br \}

http://dx.doi.org/10.5335/rbca.2015.4624

Revista Brasileira de Computação Aplicada (ISSN 2176-6649), Passo Fundo, v. 7, n. 2, p. 77-91, mai. 2015 
equipamentos do consumidor [3]. Os estudos relacionados à $Q E E$ estão se tornando cada vez mais importantes, devido às características das cargas e dos SEPs atuais, que são mais sensíveis aos distúrbios.

Dentre os vários distúrbios da $Q E E$, existe uma classe denominada “distorção harmônica". As distorções harmônicas são distorções periódicas nas formas de onda das tensões e correntes, caracterizadas pela presença de frequências que são múltiplas inteiras da frequência nominal do sistema (no caso do Brasil $60 \mathrm{~Hz}$ ), geralmente associadas à operação contínua de cargas com características não lineares [4]. Com o desenvolvimento tecnológico e da eletrônica de potência, esses distúrbios se tornam cada vez mais relevantes, devido a equipamentos sensíveis presentes nos SEPs e que exigem uma energia elétrica de melhor qualidade [5]. Alguns dos problemas causados pelas distorções harmônicas são: aquecimento excessivo de dispositivos, disparo de dispositivos de proteção[6], ressonância, tensão elevada entre neutro e terra, má operação de dispositivos e equipamentos e parada de processos produtivos [7].

Cabe ressaltar que as cargas não lineares, cada vez mais presentes nos SEPs, são as mais sensíveis aos distúrbios e também são as que mais provocam distorções harmônicas no SEP [8]. Portanto, a tendência é que a presença das harmônicas nos SEPs se agrave e aumente as perdas econômicas provocadas pela má QEE. Por esses motivos, várias pesquisas buscam métodos que melhorem a precisão e a velocidade dos algoritmos aplicados na estimação das componentes harmônicas. Dentre as diversas técnicas utilizadas, destacam-se os métodos baseados na transformada discreta de fourier (TDF)[9], transformada rápida de fourier (FFT) [10], no ajuste dos mínimos quadrados (LS) [11], na transformada wavelet [12] e no filtro de Kalman [13]. Os métodos anteriormente mencionados podem ser afetados pelo componente de corrente contínua (CCC) [10], no entanto métodos baseados em inteligência computacional, tais como redes neurais artificiais (ANN) [14] [15], otimização por enxame de partículas (Particle Swarm Optimization - PSO) [16] e Algoritmos Genéticos [17] são pouco influenciados pelo CCC [16] e apresentam bons resultados para a estimação das componentes harmônicas.

Considerando que as propostas que empregam algoritmos evolutivos $(A E)$ para a estimação de componentes harmônicas apresentam bons resultados, neste trabalho investigou-se a aplicabilidade e a eficiência do algoritmo genético compacto (CGA - compact genetic algorithm) [18]. Para efeitos de validação, foi realizada uma comparação entre o CGA e o SGA (simple genetic algorithm) [19][20]. Ambos os algoritmos foram utilizados para estimação dos valores eficazes e dos ângulos de fase das componentes harmônicas de sinais elétricos de tensão. Com a utilização do CGA, é possível obter os benefícios da utilização de AE para estimação de componentes harmônicas, contudo, utilizando um algoritmo de implementação simples, que exige poucos recursos computacionais [21].

Este artigo está dividido em mais seis seções, além desta introdução. A segunda seção contém a descrição do problema, na qual são apresentados os modelos matemáticos empregados. A terceira seção aborda os algoritmos utilizados, enfatizando descrições relativas ao algoritmo genético simples e ao algoritmo genético compacto. A quarta seção descreve a metodologia empregada para a obtenção das componentes harmônicas por meio de dois modelos matemáticos distintos. A quinta seção expõe os resultados obtidos por meio dos algoritmos empregados. A sexta seção contém discussões sobre os resultados. Por fim, a sétima seção apresenta as conclusões obtidas.

\section{Descrição do problema}

Uma onda pode ser representada matematicamente de várias maneiras. Uma delas é pela série de Fourier, na qual é possível expressar uma onda em termos de sua componente contínua, fundamental e de suas componentes harmônicas. Cada componente harmônica da onda possui sua própria amplitude e um ângulo de fase, além de uma frequência, que deve ser múltipla inteira da frequência fundamental [3]. Assim, uma forma de onda em função do tempo pode ser descrita pela Eq.(1) [16, 22]:

$$
x(t)=x_{0} e^{-\lambda t}+\sum_{i=1}^{N}\left[A_{c, i} \cos \left(i \omega_{0} t+\theta_{c, i}\right)+A_{s, i} \sin \left(i \omega_{0} t+\theta_{s, i}\right)\right]+e(t),
$$

na qual $x(t)$ é o valor resultante da soma da componente contínua com as componentes harmônicas, $x_{0}$ é a componente contínua do sinal e $\lambda$, uma constante de tempo. $A_{c, i}, A_{s, i}, \theta_{c, i}$ e $\theta_{s, i}$ são as amplitudes cosseno e seno e os ângulos de fase das $i$-ésimas harmônicas, respectivamente; $\omega_{0}$ é a frequência angular; $t$ é o tempo que ocorreu

Revista Brasileira de Computação Aplicada (ISSN 2176-6649), Passo Fundo, v. 7, n. 2, p. 77-91, mai. 2015 
a amostra; $i$ é a ordem da harmônica; e $N$ é o número de harmônicas presentes no sinal utilizado para representar $x(t)$.

Para realizar a estimação dos parâmetros, a onda é discretizada em $n$ amostras, com uma diferença constante de tempo entre as amostras. Sendo assim, para cada tempo $t_{n}$ do domínio da função haverá um valor correspondente $x\left(t_{n}\right)$. Dessa forma, a Eq.(1) pode ser reescrita conforme apontado pela Eq.(2):

$$
\left[\begin{array}{c}
x\left(t_{1}\right) \\
\vdots \\
x\left(t_{n}\right)
\end{array}\right]=[M]\left[\begin{array}{c}
x_{0} \\
A_{c, 1} \\
A_{s, 1} \\
\vdots \\
A_{c, n} \\
A_{s, n}
\end{array}\right]+\left[\begin{array}{c}
e\left(t_{1}\right) \\
e\left(t_{2}\right) \\
\vdots \\
e\left(t_{n}\right)
\end{array}\right]
$$

na qual $x\left(t_{1}\right)$ é o valor resultante da soma das componentes harmônicas para o tempo $t_{1}$, $[M]$ é a matriz representada na Eq. (3) e $e\left(t_{n}\right)$ é o erro associado a cada instante de tempo $t_{n}$ [22].

$$
[M]=\left[\begin{array}{cccccc}
e^{-\lambda t_{1}} & \cos \left(\omega_{0} t_{1}+\theta_{c, 1}\right) & \sin \left(\omega_{0} t_{1}+\theta_{c, 1}\right) & \cdots & \cos \left(N \omega_{0} t_{k}+\theta_{c, N}\right) & \sin \left(N \omega_{0} t_{k}+\theta_{c, N}\right) \\
e^{-\lambda t_{2}} & \cos \left(\omega_{0} t_{2}+\theta_{c, 1}\right) & \sin \left(\omega_{0} t_{2}+\theta_{c, 1}\right) & \cdots & \cos \left(N \omega_{0} t_{2}+\theta_{c, N}\right) & \sin \left(N \omega_{0} t_{2}+\theta_{c, N}\right) \\
\vdots & \vdots & \vdots & \vdots & \vdots & \vdots \\
e^{-\lambda t_{m}} & \cos \left(\omega_{0} t_{m}+\theta_{c, 1}\right) & \sin \left(\omega_{0} t_{m}+\theta_{c, 1}\right) & \cdots & \cos \left(N \omega_{0} t_{m}+\theta_{c, N}\right) & \sin \left(N \omega_{0} t_{m}+\theta_{c, N}\right)
\end{array}\right]
$$

Outra forma de representar a onda é por meio do somatório das formas de onda senoidais de cada componente harmônica individualmente [23], como mostrado na Eq. (4):

$$
x(t)=\sum_{i=1}^{N} A_{s, i} \sin \left(i \omega_{0} t+\theta_{s, i}\right),
$$

na qual $\mathrm{x}(\mathrm{t})$ é o valor resultante ao somar as componentes harmônicas, $A_{s, i}$ é a amplitude seno dos i-nésimos harmônicos, $\omega_{0}$ é a frequência angular, t é o tempo que durou a medida da amostra, $\theta_{s, i}$ é o ângulo de fase, i é a ordem da harmônica e $\mathrm{N}$ é o número de harmônicas presentes no sinal utilizado para representar $x(t)$.

\section{Algoritmos utilizados} trabalho.

Nesta seção será descrito o algoritmo genético simples e o algoritmo genético compacto utilizados neste

\subsection{Algoritmo genético simples}

Os AGs são algoritmos de busca e otimização global, baseados na teoria da evolução de Darwin e na genética. Os AGs partem do pressuposto que, em uma determinada população, os melhores indivíduos têm maiores chances de sobrevivência e de gerarem indivíduos cada vez mais aptos [20], garantindo, desta forma, que essa população evolua e encontre uma solução para o problema.

Conforme apresentado no pseudocódigo da Figura 1a, o algoritmo genético simples apresenta as etapas de inicialização da população, avaliação, seleção, cruzamento e mutação.

Primeiro, há a etapa de inicialização da população, na qual todos os indivíduos são avaliados utilizando uma função de avaliação. No processo de seleção, um número $N$ de indivíduos é selecionado para a etapa de cruzamento. Depois do cruzamento, os novos indivíduos gerados passam pela etapa de mutação. Em seguida, os novos indivíduos se tornam a nova população. Enquanto o critério de parada não for satisfeito, o algoritmo irá 
executar a partir da etapa de seleção. Ao fim da execução do algoritmo, a solução será o indivíduo que obtiver a melhor avaliação.

\subsection{Algoritmo genético compacto}

A própria determinação dos parâmetros necessários para o funcionamento do SGA, tais como a taxa de cruzamento e a taxa de mutação, por si só torna-se um problema de otimização dentro de outro problema de otimização. A predição do movimento das populações é consideravelmente difícil [24]. Em função disso, novos tipos de algoritmos vêm sendo desenvolvidos, os algoritmos de estimação de distribuição (EDA - Estimation of Distribution Algorithms) [24], dos quais se pode citar o CGA como um dos modelos de EDA mais simples.

O CGA é um algoritmo com eficácia semelhante à do SGA, porém, consome menos recursos computacionais por representar a população por meio de um vetor de probabilidade [21], ou seja, representa a proporção da presença de cada gene na população. Logo, por não conter uma representação física da população (apenas o vetor de probabilidade), o CGA torna-se um algoritmo mais simples de ser implementado em comparação ao SGA, além de consumir menos recursos computacionais. A Figura $1 \mathrm{~b}$ mostra o pseudocódigo do CGA, considerando como mecanismo de seleção um torneio de tamanho 2. Cabe ressaltar que o CGA não envolve a etapa de mutação, ou seja, é um algoritmo seleto-recombinativo.

O CGA tem como primeira etapa a inicialização do vetor de probabilidade, no qual para cada posição é atribuído o valor de 0.5 , ou seja, $50 \%$ de probabilidade de gerar o número 1 para aquela posição do indivíduo. A segunda etapa consiste na geração de dois indivíduos levando em consideração o vetor de probabilidade. Na terceira etapa, é realizada a avaliação dos indivíduos gerados e, na quarta etapa, ocorre a verificação de qual indivíduo obtém a melhor avaliação. Baseado na comparação entre o melhor e o pior indivíduo é realizada a atualização do vetor probabilidade na quinta etapa. Enquanto o vetor probabilidade não convergir, o algoritmo continua sua execução a partir da segunda etapa. Quando convergir, o algoritmo para sua execução e a solução é obtida por meio do vetor probabilidade.

Figura 1: Pseudocódigos dos algoritmos de otimização utilizados: (a) SGA; (b) CGA.

(a)

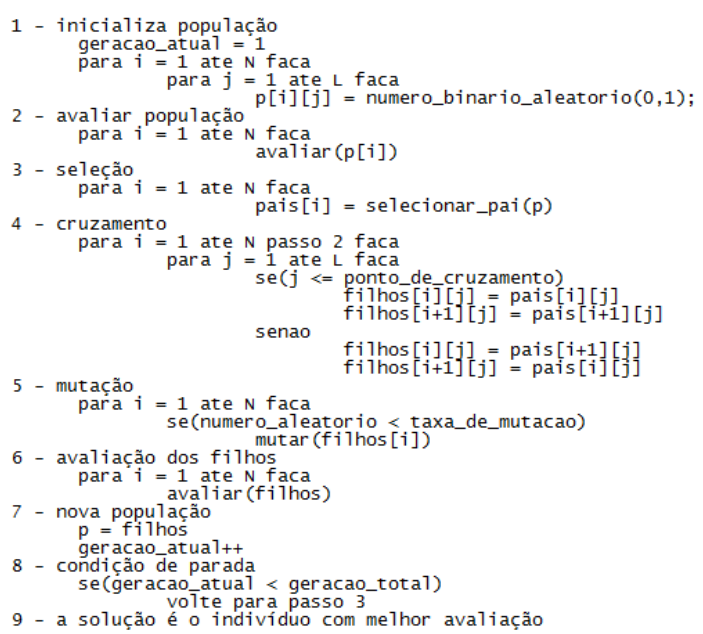

(b)

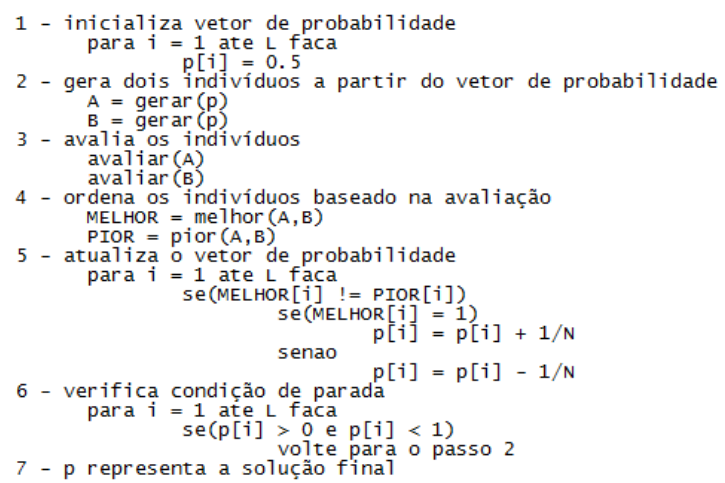

\section{Metodologia utilizada}

Para realizar a estimação de componentes harmônicas, foram utilizados os algoritmos CGA e SGA, ambos empregando os dois modelos matemáticos para estimação das componentes harmônicas previamente apresentadas. O primeiro modelo utilizando a Eq. (1) $\left(M_{1}\right)$ será implementado considerando as sete primeiras componentes da série de Fourier. Assim, o algoritmo estima trinta parâmetros, sendo 28 deles dedicados à amplitude dos senos, 
cossenos e ângulos de fase, e os outros dois para a componente contínua do sinal e para a constante de tempo. Para representar os parâmetros da série de Fourier, foram utilizados 8 bits para cada parâmetro, pois se verificou ser suficiente para representar os valores com uma boa precisão. Um indivíduo gerado quando empregado o modelo $\left(M_{1}\right)$ é representado conforme a Figura 2.

Figura 2: Representação do indivíduo segundo o modelo $M_{1}$.

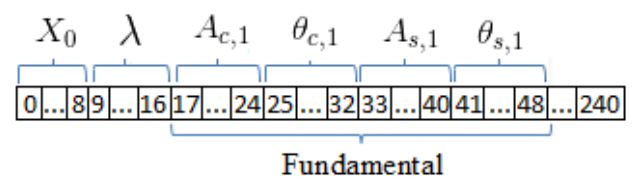

Quando é utilizado o modelo da Eq. (4) $\left(M_{2}\right)$ o algoritmo estima quatorze parâmetros, pois o $M_{2}$ estima apenas as amplitudes e fases das componentes senoidais de cada harmônica, e não a componente contínua do sinal ou a constante de tempo. Para cada um dos sete harmônicos, tem-se uma amplitude e um ângulo de fase, e dessa forma o indivíduo é representado de acordo com a Figura 3.

Figura 3: Representação do indivíduo segundo o modelo $M_{2}$.

\begin{tabular}{|c|c|c|c|c|c|c|c|c|c|c|c|c|c|}
\hline \multirow{2}{*}{\multicolumn{3}{|c|}{ Amplitude }} & \multicolumn{3}{|c|}{$\theta$} & \multicolumn{3}{|c|}{ Amplitude } & \multicolumn{3}{|c|}{$\theta$} & & \\
\hline & & & & 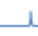 & & & 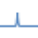 & & & $\lambda$ & & & \\
\hline 1 & $\ldots$ & 8 & 9 & $\ldots$ & 16 & 17 & $\ldots$ & 24 & 25 & $\ldots$ & 32 & $\ldots$ & 112 \\
\hline
\end{tabular}

A mesma representação dos indivíduos adotada para cada um dos modelos $\left(M_{1}\right.$ e $\left.M_{2}\right)$, foi utilizada tanto no SGA quanto no CGA. O SGA foi implementado com a taxa de cruzamento de $75 \%$. Como o CGA não contempla a etapa de mutação, com o intuito de realizar uma comparação mais justa, o SGA foi considerado sem a etapa de mutação para observar o seu processo evolutivo sem esse operador. Adotou-se $75 \%$ para a taxa de cruzamento pois foi observado em testes que uma taxa maior de cruzamento, não apresentou melhoria nos resultados, nem economia de recursos computacionais.

Os dados utilizados nos testes são valores de tensão obtidos por simulação de um sistema elétrico de potência utilizando o software ATP [25]. As avaliações dos indivíduos foram feitas por meio da comparação entre o sinal original e o sinal gerado a partir dos parâmetros estimados. Para tanto, foram gerados os sinais de acordo com (1), (4) e com a onda de referência gerada pelo ATP, contendo 64 amostras por ciclo, considerando os parâmetros estimados.

A Figura 4 ilustra a comparação entre a forma de onda de um sinal medido e a forma de onda obtida a partir dos parâmetros estimados. É possível observar que cada amostra do sinal estimado apresenta um erro em relação à amostra equivalente do sinal medido, logo, o erro total entre os sinais é obtido pela soma dos erros das amostras.

Figura 4: Erro entre a onda medida e a onda estimada.

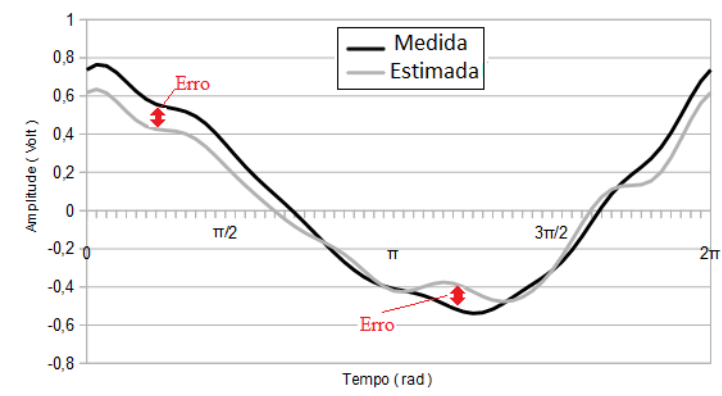

Revista Brasileira de Computação Aplicada (ISSN 2176-6649), Passo Fundo, v. 7, n. 2, p. 77-91, mai. 2015 
É possível observar que, quanto menor for o erro entre a forma de onda medida e a estimada, melhor a estimação dos parâmetros. Portanto, o objetivo é minimizar o valor da função de avaliação adotada que considera os erros entre os sinais conforme a Eq. (5):

$$
E=\sqrt{\sum_{k=1}^{N} \frac{\left(x_{m e d}\left(t_{k}\right)-x_{e s t}\left(t_{k}\right)\right)^{2}}{N}}
$$

em que $x_{m e d}$ é o sinal medido, $x_{e s t}$ é o sinal estimado, $N$ é o número de amostras em um ciclo e $E$ é o valor da função avaliação que se pretende minimizar.

Ao utilizar a representação binária para os indivíduos da população, o CGA adota critério de parada baseado na probabilidade de cada bit do indivíduo ser 1 . Logo, se em todas as posições do vetor de probabilidade a possibilidade de ser 1 for menor que 5\% ou maior que $95 \%$ o algoritmo deverá parar. Para o SGA foi adotado o mesmo critério. Considerando que se trabalha com a população em vez de um vetor de probabilidade, quando a porcentagem de bits iguais a 1 atingir 5\% ou 95\% para o mesmo lócus de todos os indivíduos da população, o SGA deverá parar. Cabe ressaltar que o torneio [20] foi o tipo de seleção utilizada.

Todos os testes com os sinais obtidos foram realizados com a população variando de 100 até 10.000 e com o tamanho de torneio variando de dois a 32 indivíduos. Para cada configuração, a média das avaliações de toda a população corresponderá ao erro médio. Cada configuração foi executada dez vezes, a fim de obter a média dos valores de erro médio, o menor erro obtido dentre as dez execuções e o número de avaliações. O número de avaliações para o SGA foi calculado como sendo o produto entre a média do número de gerações das dez execuções pelo tamanho da população. Já para o CGA, foi calculada como o produto entre o tamanho do torneio e a média do número de gerações das dez execuções.

\section{Resultados obtidos}

Os modelos e algoritmos propostos para a estimação de harmônicos foram testados, dispondo de valores de tensão obtidos por meio de simulações feitas com software ATP. Diferentes parâmetros foram variados nos algoritmos, a fim de avaliar sua influência na obtenção dos resultados. Os resultados obtidos foram comparados considerando as diferentes configurações utilizadas nos algoritmos.

\subsection{Estimação das componentes harmônicas com utilização das metodologias propostas}

No intuito de avaliar o desempenho dos algoritmos e modelos propostos, foram gerados diversos sinais com harmônicos, dispondo de um SEP simulado via software ATP. Nesta seção, será apresentada a forma de onda de uma das situações utilizadas, e essa forma de onda será comparada com as formas de onda geradas por meio dos harmônicos estimados com diferentes configurações dos algoritmos.

A Figura 5a apresenta as ondas geradas com os harmônicos estimados mediante utilização do modelo $M_{1}$ com o emprego do SGA e do CGA. Nesse teste, os parâmetros do SGA e do CGA foram: tamanho de torneio $(k)$ igual a dois e tamanho de população igual a cem indivíduos. Conforme se observa na Figura 5a, com a configuração utilizada para os AGs, o CGA apresentou uma resposta melhor para a estimação dos harmônicos, uma vez que, com a estimação proveniente do CGA, gerou-se uma onda mais próxima da onda original simulada via ATP.

A Figura $5 \mathrm{~b}$ apresenta as ondas obtidas mediante os harmônicos estimados com utilização do modelo $M_{2}$ com o emprego do SGA e do CGA. Nesse teste, os parâmetros do SGA e do CGA foram os mesmos utilizados no caso anteriormente apresentado. Conforme se observa na Figura 5b, com a configuração utilizada para os AGs, o CGA novamente apresentou uma resposta melhor para a estimação dos harmônicos, uma vez que, com a estimação proveniente do CGA, gerou-se uma onda mais próxima da onda original simulada via ATP.

Avaliando as Figuras 5a e 5b, no intuito de comparar-se o desempenho dos modelos, observa-se que o SGA utilizando $M_{2}$ obteve um resultado ligeiramente melhor do que o SGA utilizando $M_{1}$. Por sua vez, o CGA utilizando $M_{1}$ obteve uma onda mais próxima da onda medida que o CGA utilizando $M_{2}$. 
Figura 5: Ondas obtidas com parâmetros estimados pelos algoritmos considerando tamanho do torneio $(k)$ igual a 2 e tamanho de população igual a 100: (a) para $M_{1}$; (b) para $M_{2}$.

(a)

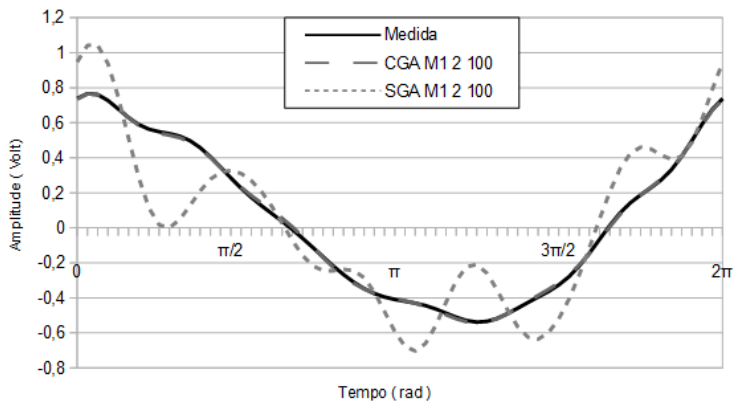

(b)

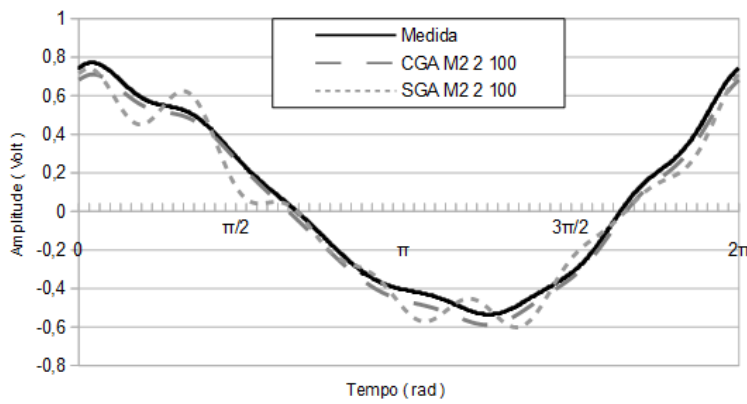

Na Figura 6a, foi utilizado o $M_{2}$, novamente com aplicação do SGA e do CGA. Pode-se perceber que o aumento do tamanho de torneio $(k)$ de dois para oito não contribuiu com a melhoria da estimação dos parâmetros, conforme observado pela diferença entre a onda estimada e medida. O aumento do tamanho de torneio $(k)$ de dois para oito permitiu uma melhoria mais visível na onda estimada pelo SGA do que pelo CGA.

Figura 6: Ondas obtidas com parâmetros estimados pelos algoritmos, adotando tamanho do torneio $(k)$ igual a 8 para $M_{2}$, considerando: (a) tamanho de população igual a 100; (b) tamanho de população igual a 500.

(a)

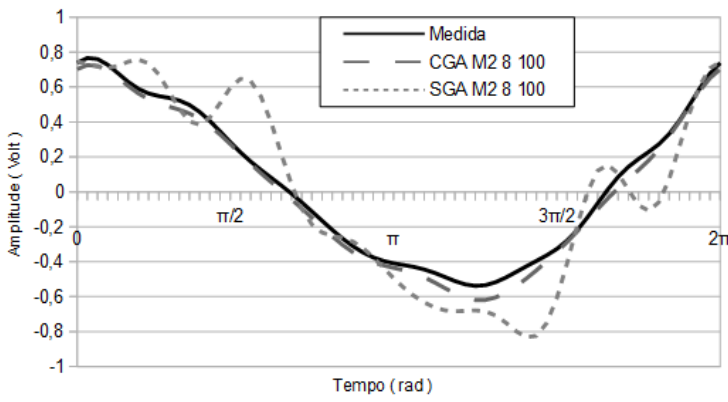

(b)

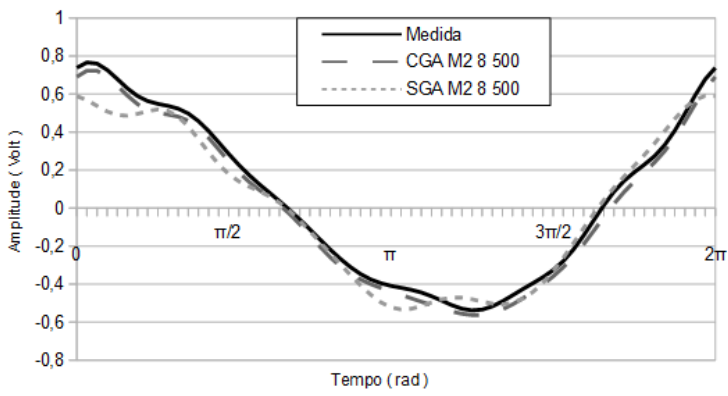

Na Figura 6b, pode-se perceber que, com o tamanho de torneio $(k)$ igual a 8 e o tamanho de população igual a quinhentos, tanto o CGA como o SGA conseguiram estimar adequadamente os parâmetros, gerando, assim, ondas próximas da onda medida. Observa-se que o aumento do tamanho da população trouxe maiores mudanças para o SGA do que para o CGA.

Para os testes apresentados pelas Figuras $6 \mathrm{a}$ e 6b, observou-se que o SGA foi mais suscetível às variações na pressão seletiva. Essa observação é válida para os demais testes realizados, conforme será discutido nas próximas seções.

As Tabelas de 1 a 4 apresentam os parâmetros de referência utilizados para obtenção de quatro formas de onda de tensão distintas. Nessas tabelas apresenta-se, também, um comparativo que envolve resultados obtidos, utilizando o CGA e o SGA, ambos adotando tanto $M_{1}$ quanto $M_{2}$ para estimação dos harmônicos, bem como a resposta gerada pela TDF. 
Tabela 1: Valor RMS da tensão da frequência fundamental $\left(V_{1}\right)$ e componentes harmônicas em termos percentuais da fundamental para a onda 1.

\begin{tabular}{lcccccc}
\hline \multicolumn{7}{c}{ Onda 1 } \\
\hline & $\begin{array}{c}\text { CGA com M1 } \\
\text { Referência }\end{array}$ & $\begin{array}{c}\text { CGA com M2 } \\
\text { Estimada }\end{array}$ & $\begin{array}{c}\text { SGA com M1 } \\
\text { Estimada }\end{array}$ & $\begin{array}{c}\text { SGA com M2 } \\
\text { Estimada }\end{array}$ & $\begin{array}{c}\text { TDF } \\
\text { Estimada }\end{array}$ \\
V1 & 0,4225 & 0,4214 & 0,4243 & 0,4514 & 0,3965 & 0,4281 \\
V2 & 12,52 & 11,87 & 9,80 & 9,95 & 9,09 & 12,06 \\
V3 & 2,59 & 3,20 & 1,96 & 7,44 & 6,29 & 2,72 \\
V4 & 4,61 & 5,27 & 4,58 & 14,47 & 4,90 & 4,58 \\
V5 & 3,58 & 2,63 & 3,27 & 40,45 & 6,29 & 3,56 \\
V6 & 5,25 & 5,17 & 4,58 & 12,52 & 3,50 & 4,65 \\
V7 & 2,00 & 1,85 & 1,96 & 12,87 & 2,10 & 1,31 \\
\hline
\end{tabular}

Tabela 2: Valor RMS da tensão da frequência fundamental $\left(V_{1}\right)$ e componentes harmônicas em termos percentuais da fundamental para a onda 2.

\begin{tabular}{|c|c|c|c|c|c|c|}
\hline & \multicolumn{6}{|c|}{ Onda 2} \\
\hline & Referência & $\begin{array}{l}\text { CGA com M1 } \\
\text { Estimada }\end{array}$ & $\begin{array}{c}\text { CGA com M2 } \\
\text { Estimada }\end{array}$ & $\begin{array}{c}\text { SGA com M1 } \\
\text { Estimada }\end{array}$ & $\begin{array}{c}\text { SGA com M2 } \\
\text { Estimada }\end{array}$ & $\begin{array}{c}\text { TDF } \\
\text { Estimada }\end{array}$ \\
\hline V1 & 0,3373 & 0,3351 & 0,3355 & 0,3770 & 0,3300 & 0,3447 \\
\hline V2 & 15,79 & 15,91 & 15,70 & 12,94 & 15,97 & 15,48 \\
\hline V3 & 6,44 & 3,34 & 5,79 & 8,94 & 12,61 & 6,71 \\
\hline V4 & 8,02 & 7,76 & 10,74 & 7,43 & 7,56 & 8,14 \\
\hline V5 & 6,36 & 6,48 & 7,44 & 4,93 & 5,88 & 6,09 \\
\hline V6 & 5,33 & 7,55 & 5,79 & 3,50 & 2,52 & 4,74 \\
\hline V7 & 4,86 & 4,95 & 5,79 & 5,23 & 4,20 & 3,73 \\
\hline
\end{tabular}

Tabela 3: Valor RMS da tensão da frequência fundamental $\left(V_{1}\right)$ e componentes harmônicas em termos percentuais da fundamental para a onda 3 .

\begin{tabular}{|c|c|c|c|c|c|c|}
\hline & \multicolumn{6}{|c|}{ Onda 3} \\
\hline & Referência & $\begin{array}{c}\text { CGA com M1 } \\
\text { Estimada }\end{array}$ & $\begin{array}{c}\text { CGA com M2 } \\
\text { Estimada }\end{array}$ & $\begin{array}{l}\text { SGA com M1 } \\
\text { Estimada }\end{array}$ & $\begin{array}{c}\text { SGA com M2 } \\
\text { Estimada }\end{array}$ & $\begin{array}{c}\text { TDF } \\
\text { Estimada }\end{array}$ \\
\hline V1 & 0,2921 & 0,2954 & 0,2856 & 0,2542 & 0,2856 & 0,2997 \\
\hline V2 & 19,99 & 20,65 & 24,27 & 43,78 & 14,56 & 19,91 \\
\hline V3 & 13,08 & 10,36 & 12,62 & 16,17 & 14,56 & 12,86 \\
\hline V4 & 8,08 & 8,02 & 2,91 & 28,56 & 8,74 & 7,76 \\
\hline V5 & 6,77 & 7,11 & 4,85 & 15,93 & 4,85 & 6,32 \\
\hline V6 & 6,53 & 6,74 & 6,80 & 14,36 & 2,91 & 5,56 \\
\hline V7 & 4,05 & 3,93 & 4,85 & 8,58 & 6,80 & 2,73 \\
\hline
\end{tabular}

Revista Brasileira de Computação Aplicada (ISSN 2176-6649), Passo Fundo, v. 7, n. 2, p. 77-91, mai. 201584 
Tabela 4: Valor RMS da tensão da frequência fundamental $\left(V_{1}\right)$ e componentes harmônicas em termos percentuais da fundamental para a onda 4 .

\begin{tabular}{ccccccc}
\hline \multicolumn{7}{c}{ Onda 4 } \\
\hline & $\begin{array}{c}\text { Onda 04 } \\
\text { Referência }\end{array}$ & $\begin{array}{c}\text { CGA com M1 } \\
\text { Estimada }\end{array}$ & $\begin{array}{c}\text { CGA com M2 } \\
\text { Estimada }\end{array}$ & $\begin{array}{c}\text { SGA com M1 } \\
\text { Estimada }\end{array}$ & $\begin{array}{c}\text { SGA com M2 } \\
\text { Estimada }\end{array}$ & $\begin{array}{c}\text { TDF } \\
\text { Estimada }\end{array}$ \\
V1 & 0,7122 & 0,7096 & 0,7071 & 0,6748 & 0,6794 & 0,7124 \\
V2 & 2,79 & 2,21 & 2,75 & 11,68 & 3,67 & 2,78 \\
V3 & 2,09 & 2,03 & 1,96 & 4,30 & 5,31 & 2,09 \\
V4 & 1,73 & 1,11 & 1,96 & 3,11 & 3,67 & 1,73 \\
V5 & 1,57 & 2,06 & 1,96 & 9,93 & 2,86 & 1,57 \\
V6 & 1,61 & 1,76 & 1,18 & 8,30 & 0,41 & 1,61 \\
V7 & 2,29 & 2,18 & 1,96 & 1,87 & 2,86 & 2,27 \\
\hline
\end{tabular}

Cabe comentar que os resultados reportados nas tabelas de 1 a 4 com utilização do CGA foram obtidos considerando o tamanho da população igual a cem indivíduos e com o torneio de tamanho dois. Já os resultados, reportados nas quatro tabelas, com utilização do SGA foram obtidos considerando o tamanho da população igual a quinhentos indivíduos e com o torneio de tamanho 8. Ressalta-se que os valores apresentados são sempre muito próximos aos de referência, sendo, inclusive, em muitos casos, mais próximos do que os obtidos pela TDF.

Observa-se que em algumas situações, a estimação realizada com uso da TDF foi mais precisa do que a obtida com os demais algoritmos, contudo, em aplicações em que existam recursos computacionais abundantes, outras configurações dos AGs podem ser empregadas, possibilitando obtenção de resultados ainda melhores que os apresentados nas tabelas de 1 a 4 .

Nas seções a seguir, discutem-se os resultados obtidos, de uma forma mais geral, analisando-se alguns critérios de qualidade das soluções encontradas.

\subsection{Observação do erro médio}

O erro médio (dado pela média entre os erros de todas as soluções após uma execução do algoritmo) foi observado com o intuito de avaliar a convergência dos algoritmos. Um erro médio baixo significa que todas as soluções estão convergindo para a mesma região do espaço de busca, o que implica uma boa robustez do método.

Para o tamanho do torneio igual a dois, conforme a Figura 7a, o $S G A$ e $C G A$ utilizando $M_{1}$, tiveram o erro médio semelhante a partir do tamanho de população igual a 2 mil indivíduos, sendo que este permaneceu praticamente constante para as execuções com populações de até 10 mil indivíduos. O CGA utilizando $M_{2}$ teve um erro médio maior que as demais configurações. Com o aumento do tamanho da população o SGA, utilizando $M_{1}$, obteve um erro médio melhor que o $C G A$ e que o $S G A$ quando utilizado o $M_{2}$. Nesse cenário o $C G A$ utilizando $M_{1}$ obteve o menor erro médio para todos os tamanhos de população.

Considerando o tamanho do torneio igual a 8, conforme se observa na Figura $7 \mathrm{~b}$, inicialmente o SGA utilizando $M_{1}$ teve erro médio superior às demais situações. Porém, com o aumento da população, o erro médio do SGA utilizando $M_{1}$ ficou próximo ao do CGA e SGA, que utilizaram $M_{2}$. O CGA utilizando $M_{1}$ teve o erro médio inferior ao dos demais gráficos.

Para o tamanho do torneio igual a 32, conforme a Figura 7c, o erro médio do SGA utilizando $M_{1}$ foi superior aos demais para todos os tamanhos de população analisados. O CGA utilizando $M_{2}$ teve o erro praticamente constante, e menor que os gráficos dos SGAs para ambas as formas da série de Fourier. O CGA com $M_{1}$ teve o menor erro do cenário analisado.

Quando se analisa o erro médio considerando a utilização do mesmo modelo com aplicação de algoritmos distintos, percebe-se que o desempenho é bem semelhante. O mesmo vale para quando se observa a aplicação do mesmo algoritmo aos modelos diferentes, que têm comportamento similar quanto ao erro médio. Somente na situação em que se adotou 32 como tamanho do torneio, observou-se certa discrepância entre o comportamento do SGA e do CGA quanto ao erro médio. O erro médio observado com o CGA foi sempre menor do que o do 
Figura 7: Comparação do erro médio obtido com utilização do $C G A$ e do $S G A$ considerando $M_{2}$ e $M_{1}$, para: (a) tamanho do torneio igual a 2; (b) tamanho do torneio igual a 8; (c) tamanho do torneio igual a 32.

(a)

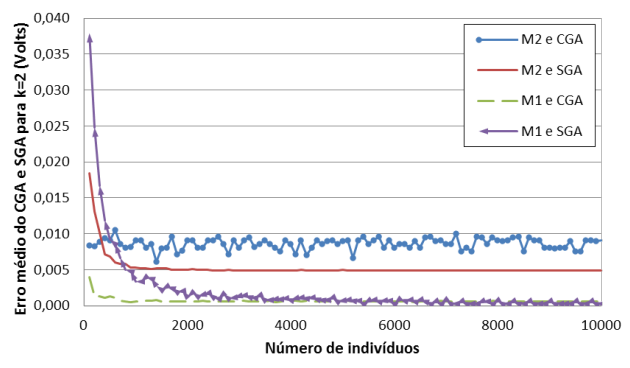

(b)

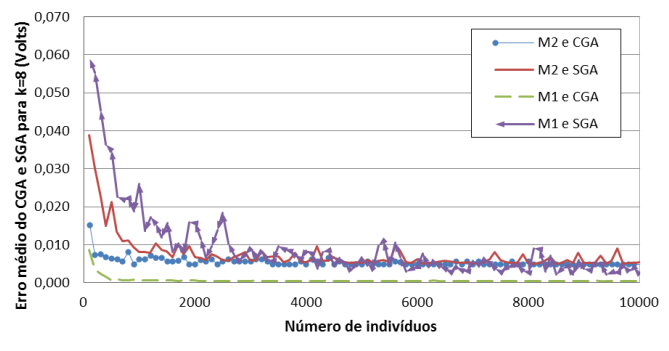

(c)

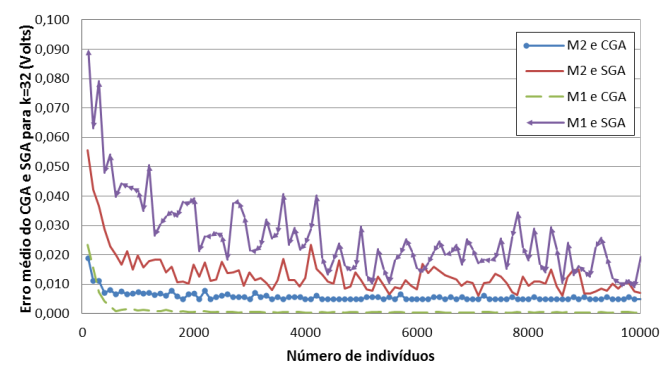

SGA para os diversos tamanhos de população adotados. Como o $M_{1}$ possui mais parâmetros a serem estimados, o aumento da pressão seletiva influenciou de forma negativa a convergência do SGA.

\subsection{Observação do menor erro}

Foi avaliado o menor erro obtido durante cada execução do algoritmo, para se verificar em que situações os algoritmos permitiam a obtenção das melhores respostas e com que frequência elas eram obtidas. Considerando o tamanho do torneio igual a dois, conforme a Figura 8a, o SGA com $M_{1}$ apresentou um erro com valor superior aos demais nos menores tamanhos de população. A partir do tamanho de população igual a 2 mil, obteve-se o erro praticamente igual ao do CGA com $M_{1}$. O CGA e o SGA com $M_{2}$ obtiveram erros semelhantes em quase todos os tamanhos de população.

Para o tamanho do torneio igual a 8, conforme a Figura 8b, o erro do SGA com $M_{2}$ diminuiu com o aumento do tamanho da população e se estabilizou a partir do tamanho de população igual a 2 mil, igualando-se com o CGA utilizando $M_{2}$. O SGA com $M_{1}$ tem o erro maior do que os demais a partir do tamanho de população igual a 2.100 . A partir do tamanho de população igual a 2.100, $M_{1}$ utilizando o SGA apresenta erro com valor inferior ao CGA e ao SGA com ambos utilizando $M_{2}$. O CGA com $M_{1}$ possui valores de erro sempre menores que os demais casos.

Com o tamanho do torneio igual a 32, conforme a Figura 8c, o SGA com $M_{2}$ apresenta o valor do erro superior ao CGA com $M_{2}$, porém, igualam-se no tamanho de população (4.300) e estabilizam=se. O SGA com $M_{1}$ tem o menor erro, maior que os demais, porém, a partir do tamanho 4.300, para alguns tamanhos de população, seu menor erro apresenta valores inferiores ao CGA e ao SGA com $M_{2}$. O CGA com $M_{1}$ possui valores de menor erro melhores que os demais casos para todos os tamanhos de população.

Com essa análise, é possível afirmar que populações com tamanhos maiores que mil indivíduos não agregam melhorias quanto à qualidade das soluções obtidas para o problema em questão, independentemente do modelo e do algoritmo adotados. Comparando-se os gráficos de erro médio e de menor erro, é possível concluir que as

Revista Brasileira de Computação Aplicada (ISSN 2176-6649), Passo Fundo, v. 7, n. 2, p. 77-91, mai. 201586 
populações dos diversos testes realizados estão convergindo para a melhor solução, pois os gráficos de erro médio são muito semelhantes aos de menor erro, o que implica dizer que as soluções da população estão convergindo para regiões próximas no espaço de busca.

Figura 8: Comparação do menor erro obtido com utilização do CGA e do SGA, considerando $M_{2}$ e $M_{1}$, para: (a) tamanho do torneio igual a 2; (b) tamanho do torneio igual a 8; (c) tamanho do torneio igual a 32.
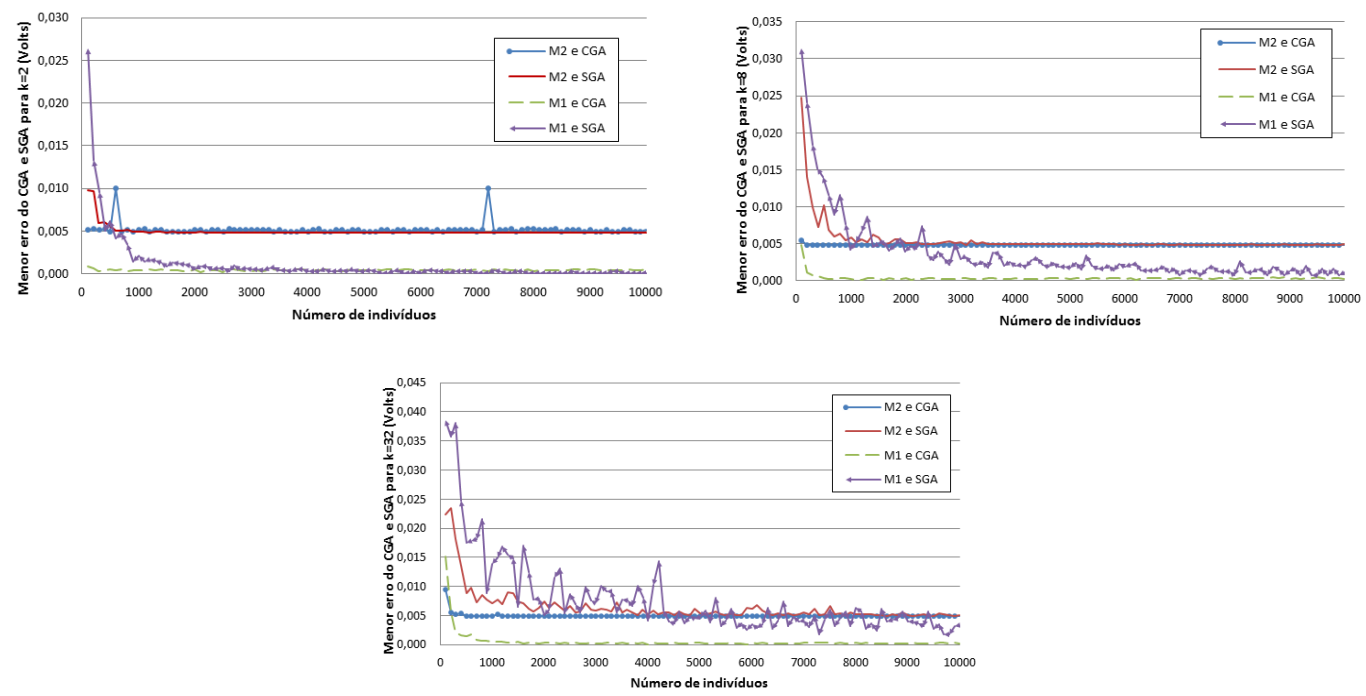

\subsection{Observação do número de avaliações}

Por meio do número de avaliações da função objetivo, é possível avaliar o tempo de convergência dos modelos e algoritmos adotados. Essa análise é muito importante para determinação da viabilidade do uso desses modelos na prática.

O CGA com $M_{1}$, para todos os tamanhos de torneio, obteve o maior número de avaliações até que o algoritmo convergisse, conforme pode ser observado nas Figuras 9a, 9b e 9c.

O SGA e CGA, com $M_{2}$, apresentaram os menores números de avaliações com o tamanho de torneio $(k)$ igual a dois, em que o SGA apresentou valores menores de que o CGA. Com o tamanho de torneio $(k)$ igual a 8, Figura 9b, o SGA utilizando $M_{1}, M_{2}$ e o CGA utilizando $M_{2}$ apresentaram resultados semelhantes, mas o SGA apresentou os menores valores utilizando $M_{2}$. Para tamanho de torneio igual a 32, Figura 9c, o SGA utilizando os dois modelos apresentou os menores resultados, e o CGA utilizando $M_{2}$ apresentou valores menores apenas que o CGA utilizando $M_{1}$.

É possível observar que a relação entre o número de avaliações realizadas pelo CGA para o $M_{1}$ e $M_{2}$, à medida que se aumenta a população, mantém-se constante independentemente do tamanho do torneio adotado. Verifica-se, também, que o $M_{2}$ sempre requer um menor número de avaliações para convergir. Além disso, cabe ressaltar que o $M_{1}$ é mais complexo e apresenta mais parâmetros, o que demanda mais tempo de computação em cada avaliação da função objetivo. 
Figura 9: Número de avaliações obtido com utilização do CGA e do SGA, considerando $M_{2}$ e $M_{1}$, para: (a) tamanho do torneio igual a 2; (b) tamanho do torneio igual a 8; (c) tamanho do torneio igual a 32.

(a)

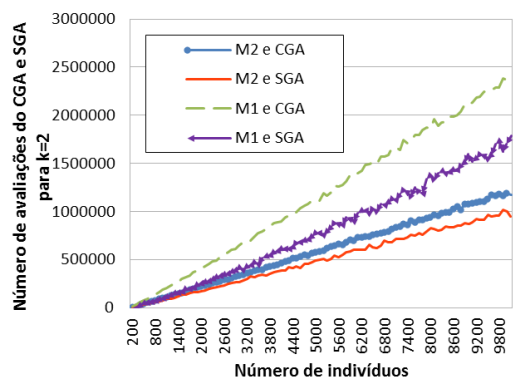

(b)

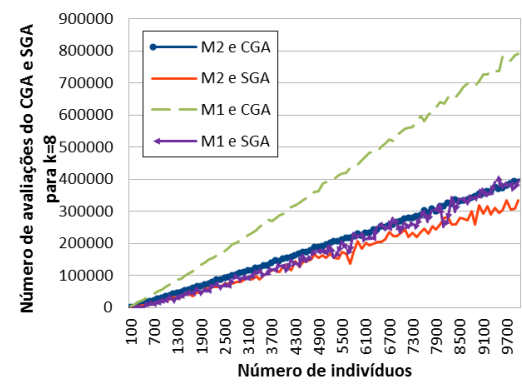

(c)

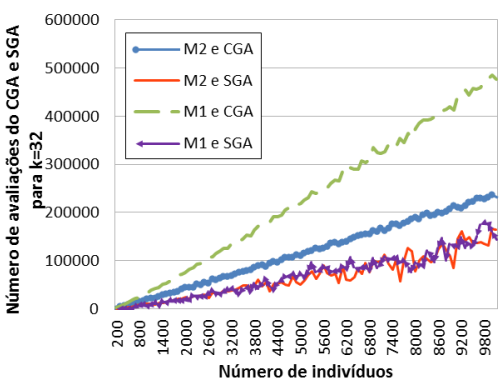

\section{Discussões}

Após a apresentação dos resultados e testes realizados, algumas constatações merecem ser destacadas. Inicialmente, quanto ao erro médio com torneio de tamanho 8, observa-se que o CGA obtém resultados melhores que o SGA, utilizando tanto o $M_{1}$ como o $M_{2}$.

Já para os gráficos do menor erro, considerando o mesmo tamanho de torneio, igual a oito, o CGA utilizando o $M_{1}$ apresenta um erro menor em relação ao apresentado pelo SGA em qualquer tamanho de população. Por sua vez, o CGA utilizando o $M_{2}$ apresenta resultados semelhantes aos obtidos com o SGA com ambos os modelos.

No que tange ao número de avaliações, para um tamanho de torneio igual a oito, o número de avaliações do CGA com utilização de $M_{2}$ tende a ser similar ao do SGA, independentemente se o modelo utilizado é o $M_{1}$ ou o $M_{2}$. Além disso, o número de avaliações do CGA com utilização de $M_{2}$ tende a ser metade do número de avaliações do CGA com utilização de $M_{1}$.

Dessa forma, é possível afirmar que a utilização do $M_{2}$, com populações menores que mil indivíduos e com tamanho de torneio igual a oito, é capaz de solucionar adequadamente o problema apresentado. Contudo, considerando a possibilidade de uma aplicação embarcada, e observando-se os valores de erros de estimação obtidos nos testes, destaca-se que é possível a utilização do $M_{2}$ com torneio de tamanho dois e população de cem indivíduos para obtenção de resultados satisfatórios. Essa última configuração foi a adotada nos resultados apresentados com utilização do CGA nas Tabelas de 1 a 5 e permanecerá sendo empregada em futuros estudos de implementação embarcada em FPGA das metodologias aqui apresentadas. 


\section{Conclusões}

As distorções harmônicas são distorções periódicas nas formas de onda das tensões e correntes, caracterizadas pela presença de frequências que são múltiplas inteiras da frequência nominal do sistema, geralmente associadas à operação contínua de cargas com características não lineares. A importância deste estudo se deve ao fato de as harmônicas interferirem no funcionamento de equipamentos eletrônicos, até mesmo chegando a danificálos, portanto, esse distúrbio deve ser identificado e diagnosticado com rapidez e precisão, possibilitando a adoção de medidas que venham a saná-lo. Nesse sentido, este artigo apresenta a aplicação do algoritmo genético simples e do algoritmo genético compacto para a estimação de componentes harmônicas.

Os sinais utilizados nas simulações foram gerados pelo software ATP e discretizados em 64 amostras por ciclo. As amplitudes e fases da série de Fourier nos modelos $M_{1}$ e $M_{2}$ são estimadas pelos algoritmos CGA e SGA para gerar um sinal de onda contendo as distorções harmônicas. Posteriormente, é calculado o erro médio quadrático entre cada uma das 64 amostras de ambas as ondas, comparando a onda obtida pelo uso dos algoritmos evolutivos e a onda obtida via simulação no software ATP. Para cada tamanho de população e tamanho de torneio, a fim de validar os resultados, os algoritmos foram executados dez vezes e obtidos os valores de: erro médio, menor erro e número de avaliações. Os resultados encontrados permitem inferir sobre qual algoritmo apresenta um melhor comportamento a partir dos ajustes dos parâmetros tamanho de população e tamanho do torneio.

Para saber qual algoritmo melhor se comporta diante de uma maior ou menor disponibilidade de recursos computacionais, foram feitos testes variando o tamanho de população e o tamanho do torneio. O tamanho de população variou entre cem e 10 mil, a fim de analisar o comportamento dos algoritmos diante de diferentes situações e de verificar com qual tamanho de população é possível conseguir resultados viáveis, levando em consideração que o aumento da população implica no aumento do tempo de execução do algoritmo. No procedimento de seleção, o torneio foi realizado adotando-se tamanhos de 2, 4, 8, 16 e 32 indivíduos, para observar a influência na pressão seletiva no algoritmo. Com os resultados encontrados, verificou-se que os dois algoritmos apresentam soluções adequadas para o problema em questão, desde que sejam adotados um modelo e parâmetros convenientes. Importa destacar que, quando se utiliza o tamanho de população igual a cem e tamanho de torneio igual a dois indivíduos, o CGA passa a ter um erro inferior ao SGA para a mesma configuração. Um maior número de gerações é necessário quando se utiliza o $M_{1}$ em razão de haver mais parâmetros para otimizar quando comparado com o $M_{2}$. Portanto, para situações reais que exigem um menor esforço computacional o CGA, utilizando o $M_{2}$ com tamanho do torneio igual a dois indivíduos e tamanho de população igual a cem é suficiente para a obtenção de resultados satisfatórios.

O estudo apresentado mostrou gráficos do erro médio, do menor erro e do número de avaliações em função do tamanho de população. Também foram apresentados gráficos comparando uma onda de referência com ondas obtidas ao utilizar o CGA e o SGA nos modelos $M 1$ e $M 2$. Para o CGA, utilizando tamanho de torneio igual a dois e tamanho de população igual a cem, a onda obtida foi mais próxima da onda de referência que o SGA, para tamanho de torneio igual a oito e tamanho de população igual a quinhentos. Constatou-se, também, que, para tamanho de população igual a cem e utilizando o número de avaliações tanto do CGA quanto do SGA, essa onde fica bastante próxima. Conforme mostrado neste trabalho, o CGA apresentou resultados tão bons quanto o SGA, porém, por utilizar menos recursos computacionais, há possibilidade de, em trabalhos futuros, aproveitar o forte paralelismo presente no algoritmo genético para implementá-lo em um sistema embarcado.

\section{Agradecimentos}

Os autores agradecem o apoio da Fundação de Amparo à Pesquisa do Estado do Piauí (Fapepi), do Centro de Tecnologia e Urbanismo (CTU) da Universidade Estadual do Piauí (UESPI) e do Departamento de Computação (DC) da Universidade Federal do Piauí (UFPI).

\section{Referências}

1 OLIVEIRA, C. C. B. de et al. Introducão a sistemas elétricos de potência: componentes simétricas. [S.1.]: Edgard Blücher, 2005.

Revista Brasileira de Computação Aplicada (ISSN 2176-6649), Passo Fundo, v. 7, n. 2, p. 77-91, mai. 2015 
3 DUGAN, R. C.; MCGRANAGHAN, M. F.; BEATY, H. W. Electrical power systems quality. New York, NY: McGraw-Hill, I c1996, v. 1, 1996.

4 GALHARDO, M. A. B.; PINHO, J. T. Modelagem de cargas não-lineares em sistemas elétricos de potência. V SBQEE-Seminário Brasileiro sobre Qualidade de Energia Elétrica, Aracajú, 2003.

5 SHORT, T. A. Distribution reliability and power quality. [S.1.]: CRC Press, 2005.

6 MAIA, R. M. Estudo de Caso de Afundamentos de Tensão nos Componentes do Sistema Elétrico Industrial da Moinhos Vera Cruz. Monografia presentada em el curso de Especialización en Automatización Industrial de La Universidad Federal de Minas Gerais, 2008.

7 MORENO, H. Harmônicas nas instalações elétricas: causas, efeitos e soluções. [S.1.]: Procobre, 2001.

8 ARRILlaGA, J.; SMITH, B. C. . W. N. R. . W. A. R. Power system harmonic analysis. [S.1.]: Wiley, 1997.

9 ZHANG, F.; GENG, Z.; YUAN, W. The algorithm of interpolating windowed FFT for harmonic analysis of electric power system. Power Delivery, IEEE Transactions on, IEEE, v. 16, n. 2, p. 160-164, 2001.

10 GIRGIS, A. A.; CHANG, W. B.; MAKRAM, E. B. A digital recursive measurement scheme for online tracking of power system harmonics. IEEE Transactions on Power Delivery, IEEE, v. 6, n. 3, p. 1153-1160, 1991.

11 KAMWA, I.; GRONDIN, R. Fast adaptive schemes for tracking voltage phasor and local frequency in power transmission and distribution systems. In: IEEE. Transmission and Distribution Conference, 1991., Proceedings of the 1991 IEEE Power Engineering Society. [S.1.], 1991. p. 930-936.

12 WILKINSON, W. A.; COX, M. Discrete wavelet analysis of power system transients. Power Systems, IEEE Transactions on, IEEE, v. 11, n. 4, p. 2038-2044, 1996.

13 CHEN, C. et al. Extended real model of Kalman filter for time-varying harmonics estimation. IEEE Transactions on Power Delivery, IEEE, v. 25, n. 1, p. 17-26, 2010.

14 LIN, H. C. Intelligent neural network-based fast power system harmonic detection. IEEE Transactions on Industrial Electronics, IEEE, v. 54, n. 1, p. 43-52, 2007.

15 VARADAN, S.; MAKRAM, E. Practical considerations in the application of neural networks to the identification of harmonic loads. Electric Power Systems Research, Elsevier, v. 30, n. 2, p. 103-106, 1994.

16 RABELO, D. A.; LEMOS, M. V.; BARBOSA, D. Power system harmonics estimation using Particle Swarm Optimization. In: IEEE. Evolutionary Computation (CEC), 2012 IEEE Congress on. [S.1.], 2012. p. 1-6.

17 MACÊDO, R. A. et al. A new technique based on genetic algorithms for tracking of power system harmonics. In: IEEE. Neural Networks, 2002. SBRN 2002. Proceedings. VII Brazilian Symposium on. [S.1.], 2002. p. 712.

18 PELIKAN, M.; GOLDBERG, D. E.; LOBO, F. G. A survey of optimization by building and using probabilistic models. Computational optimization and applications, Springer, v. 21, n. 1, p. 5-20, 2002.

19 COLEY, D. A. An introduction to genetic algorithms for scientists and engineers. [S.1.]: World scientific, 1999.

20 HOLLAND, J. H. Adaptation in natural and artificial systems: An introductory analysis with applications to biology, control, and artificial intelligence. [S.1.]: U Michigan Press, 1975.

21 HARIK, G. R.; LOBO, F. G.; GOLDBERG, D. E. The compact genetic algorithm. IEEE Transactions on Evolutionary Computation, IEEE, v. 3, n. 4, p. 287-297, 1999.

22 SEIFOSSADAT, S. G. et al. Harmonic estimation in power systems using adaptive perceptrons based on a genetic algorithm. WSEAS Transactions On Power Systems, n. 11, 2007. 
23 HAMID, E. Y.; KAWASAKI, Z.-I.; MARDIANA, R. Wavelet packet transform for RMS and power measurements. In: IEEE. Power Engineering Society Summer Meeting, 2001. [S.1.], 2001. v. 2, p. 1243-1245.

24 LARRANAGA, P. A review on estimation of distribution algorithms. In: Estimation of distribution algorithms. [S.1.]: Springer, 2002. p. 57-100.

25 MEYER, W. S.; LIU, T.-h. Alternative transients program (ATP) rule book. Canadian/American EMTP User Group, v. 2000, p. 5-2, 1987.

Revista Brasileira de Computação Aplicada (ISSN 2176-6649), Passo Fundo, v. 7, n. 2, p. 77-91, mai. 201591 